\title{
A Matheuristic Approach to Integrate Humping and Pullout Sequencing Operations at Railroad Hump Yards
}

Haahr, Jørgen Thorlund; Lusby, Richard Martin

Published in:

Networks

Link to article, DOI:

$10.1002 /$ net.21665

Publication date:

2016

Document Version

Peer reviewed version

Link back to DTU Orbit

Citation (APA):

Haahr, J. T., \& Lusby, R. M. (2016). A Matheuristic Approach to Integrate Humping and Pullout Sequencing Operations at Railroad Hump Yards. Networks, 67(2), 126-138. https://doi.org/10.1002/net.21665

\section{General rights}

Copyright and moral rights for the publications made accessible in the public portal are retained by the authors and/or other copyright owners and it is a condition of accessing publications that users recognise and abide by the legal requirements associated with these rights.

- Users may download and print one copy of any publication from the public portal for the purpose of private study or research.

- You may not further distribute the material or use it for any profit-making activity or commercial gain

- You may freely distribute the URL identifying the publication in the public portal 


\title{
A Matheuristic Approach to Integrate Humping and Pullout Sequencing Operations at Railroad Hump Yards
}

\author{
Department of Engineering Management \\ Technical University of Denmark \\ Jørgen Thorlund Haahr and Richard Martin Lusby
}

October 23,2015

\begin{abstract}
This paper presents a novel matheuristic for solving the Hump Yard Block-to-Track Assignment Problem. This is an important problem arising in the railway freight industry and involves scheduling the transitions of a set of rail cars from a set of inbound trains to a set of outbound trains over a certain planning horizon. It was also the topic of the 2014 challenge organised by the Railway Applications Section of the Institute for Operations Research and the Management Sciences for which the proposed matheuristic was awarded first prize. Our approach decomposes the problem into three highly dependent subproblems. Optimization-based strategies are adopted for two of these, while the third is solved using a greedy heuristic. We demonstrate the efficiency of the complete framework on the official datasets, where solutions within $4-14 \%$ of a known lower bound (to a relaxed problem) are found. We further show that improvements of around $8 \%$ can be achieved if outbound trains are allowed to be delayed by up to two hours in the hope of ensuring an earlier connection for some of the rail cars.
\end{abstract}

Keywords - Freight Train Optimization, Hump Yard Optimization, Block To Track Assignment, Matheuristic, Mixed Integer Programming, Rolling Horizon

\section{Introduction}

This paper addresses the 2014 challenge posed by the Railway Applications Section (RAS) of the Institute for Operations Research and the Management Sciences. The challenge focuses on an important problem arising in the railway freight transportation industry known as the Hump Yard Block-to-Track Assignment (HYBA). In particular, the problem requires one to consider, and schedule, the movements of a number of rail cars through a classification or hump yard. The primary purpose of such a yard is to act as a consolidation point, where rail cars arriving over a certain time horizon on a number of inbound trains are rearranged, or classified, into groups of rail cars sharing the same destination. These groups are then subsequently pulled out and combined to form new outbound trains, which remove rail cars from the classification yard. A classification yard typically consists of four main components: an arrival yard, a hump, a classification bowl, and a departure yard. During the processing of an inbound train each of its 
rail cars is pushed over the hump, and, under the influence of gravity, and with the use of switches, rolls to a pre-assigned classification track in the bowl. Bowls typically consist of multiple, parallel tracks of possibly different lengths, where partial outbound trains can be assembled before being pulled together to form outbound trains in the departure yard.

How to handle the steady flow of rail cars is of paramount importance to the efficiency of any classification yard. However, coordinating the processing of arriving inbound trains with the allocation of classification tracks and the assembly of outbound trains is not a trivial task. All processes within the yard are subject to a variety of different restrictions and, if scheduled poorly, can result in a situation where rail cars needlessly wait long periods of time before leaving on an outbound train. The aim of the HYBA is to process all cars in such a way that their average dwell time in the yard is minimized.

In this paper we present a novel solution approach for the HYBA problem. We propose a matheuristic based on a decomposition of the problem into three distinct, highly dependent subproblems. A matheuris-

tic embeds mathematical programming techniques within a larger heuristic framework. For two of the subproblems exact optimization-based solution strategies are employed. These are coupled with a heuristic approach to the third, and the performance of the full heuristic is analysed using the official data sets. These data sets are of a practical size and are based on a typical North American classification yard; each considers a planning horizon of 42 days during which 702 inbound trains arrive (carrying in total 52,246 rail cars) to be processed. There are between 42 and 58 classification tracks on which to sort the rail cars, which comprise 46 different destinations. Furthermore, there are 18 daily outbound trains. An outbound train is scheduled to depart at the same time every day, and each destination is served by exactly one outbound train. On such data sets, the proposed approach obtains acceptable solutions, within 4-14\% of a known lower bound (for a relaxed problem), for all data sets. We report run-times of at most 11 minutes.

To identify any bottlenecks at the considered classification yard we also perform a series of what-if analyses. For example, we discuss the effects of having longer classification tracks or more capacity on the outbound trains and compare the performance improvement to the base case. Finally, we show that substantial reductions of around $8 \%$ in average dwell time can be made if it is possible to allow outbound trains to be delayed by up to two hours. Given the relatively short run times of the approach, it is clearly evident that the proposed methodology is equally applicable at the strategic level planning decisions concerning railway classification yard design.

In what follows we describe aspects of the solution approach in more detail. We begin in Section 2 with a short summary of previous research in this area. Section 3 provides a formal description of the problem, while Section 4 introduces the models developed, together with their respective solution approaches. The performance of the approach is the subject of Section 5, where we present results on the benchmark instances provided. Finally, conclusions are drawn in Section 6.

\section{$2 \quad$ Literature Review}

The highly complex nature of hump yard planning makes it a perfect application for operations research methodologies. Not surprisingly, various studies have been conducted on problems similar in nature, but not identical, to the one considered in this paper. In this section we provide a brief review of the research that is available in the literature and, where relevant, point out any differences from the problem at hand. 
Furthermore, we restrict the review to those studies that concern hump yard planning on a microscopic level only (i.e., planning the shunting movements within a single hump yard) since this is precisely the problem we address in this paper. For a general introduction to shunting within hump yards the reader is referred to $[6,10]$, while models that address hump yard planning at a macroscopic level (i.e., between different yards), commonly known as the railroad blocking problem, can be found in $[1,2,18]$.

Methods for hump yard planning are typically categorized as being either single or multi stage methods, with the latter being by far the more researched. In a single stage method, each rail car arriving on an inbound train can be humped to a classification track exactly once before it is pulled out to an outbound train. Multi stage methods, on the other hand, allow rehumping (i.e., cars can be humped multiple times). This additional flexibility is provided in the hope of obtaining a better sorting of the cars and, ultimately, a more efficient use of the yard. Typically a so-called mixing track is specified, where any car assigned to the mixing track can be pulled back to the hump and classified again.

The works $[3,4,5]$ specifically address the problems of sorting and classifying rail cars at a hump yard using the mixed track concept. All papers restrict their attention to scheduling the classification bowl of the hump yard only. A noticeable difference between this problem and the one that we consider is that the formation of the outbound trains happens directly on the tracks of the classification bowl. Typically a track of the bowl is dedicated to a specific outbound train and the cars destined for the outbound train appear on the train in the order they are humped to the track. As reserving a classification track for a single outbound train claims significant sorting capacity, it is impractical to allocate an outbound train an entire track from the arrival of its first car until its departure. As such, a number of mixing tracks is used to temporarily hold cars for different trains. The cars on such tracks are then rehumped.

In [3], the authors describe an integer programming formulation that attempts to minimize the number of rehumpings that must be performed. These extra shunting movements are termed roll-ins by the authors. The model is solved using a branch-and-price algorithm and tested on 192 real-life instances from the Hallsberg marshalling (hump) yard in Sweden. The authors provide a direct comparison with a compact integer programming formulation and demonstrate the superiority of the column generation procedure. Reference [4] extends this methodology to model situations in which the arriving rail cars each belong to a certain block, and these blocks must appear in a pre-specified order on the outbound train. Again, the Hallsberg hump yard in Sweden forms the basis of the computational study, where 50 instances with a planning horizon of three days are considered. An extension to [3] is also considered in [5], where a new arc-based model is presented, along with a rolling-horizon solution framework and an analysis of yard capacity.

The problem we consider shares strong similarities with that considered in $[3,4,5]$; however, there are several key differences. First, the incoming sequence of cars is not fixed in our problem (i.e., we can decide the order in which to process the inbound trains), we are not allowed to rehump cars, and we must coordinate the assembly of outbound trains using a set of pullback engines. That is, the assignment of a rail car to a classification track does not implicitly indicate the outbound train, nor the order in which it appears on an outbound train.

Identifying an efficient sorting of inbound rail cars at classification yards is also the focus of [8]. The authors adopt a more theoretical approach to the problem and prove that the problem of finding the minimum number of tracks required to sort a set of arriving rail cars into blocks of cars that can be 
pulled out in a specific order from the classification tracks is NP-complete. This topic is also addressed by $[7,12,17]$. In [12], the authors develop a novel encoding strategy for classification schedules, discuss its complexity, and present algorithms that can be used to solve practical rail car classification problems. Theoretical aspects of rail car classification are also considered in [17]. In addition, the author describes several practical extensions of the problem, and an integer programming formulation is developed to solve the classification problem. Finally, [7] completes an open proof from [12] and show that identifying optimal classification schedules in constructing one long outbound train from multiple inbound trains is NP-Hard.

Dirnberger and Barkan [9] consider improving the performance of classification yards and introduce the concept of so-called lean railroading. This approach adapts production management strategies to the railroad environment. The pull-down, or outbound train assembly, process is identified as the main bottleneck, and the authors suggest that to improve the performance of classification yards emphasis should be placed on identifying quality sorting strategies instead of merely measuring the number of cars processed at the hump. Studies reported in the paper suggest that capacity for train assembly can be increased by as much as $26 \%$.

Attempts to improve the connection reliability of hump yards are provided in the two part series of papers $[13,14]$. Determining which cars to process at the hump, and in which order, is critical in ensuring that cars meet specific (i.e., the earliest) outbound departures. The first paper [13] considers the relationship between priority-based-classification and dynamic car scheduling to produce a reliable service. The author emphasizes the need for better information to be available at the time at which a car is humped (ideally the outbound train to which it will be assigned). This can be coupled with a more efficient block-to-track assignment to ensure that the classification yard is being used to its full potential. This is precisely the topic of the second paper [14]. The author describes a dynamic car block-to-track assignment strategy based on delivery commitment rather than a first-in-first-out strategy. In other words, cars with very little schedule slack should have access to the first available outbound train capacity. The proposed heuristic framework sorts cars by outbound train and destination yard block as opposed to just destination yard block, giving greater knowledge regarding the exact make-up of each outbound train.

He et al. [11] present a Mixed Integer Program (MIP) model for optimizing single stage hump yard operations (i.e., no rehumping), from inbound train classification to outbound train assembly and departure. The model also appears to account for outbound block standing orders and limits on the number of pullback engines available to do the sorting. Due to its size and complexity, the authors present a decomposition-based heuristic and discuss its performance on several practical instances arising in China. The problems considered up to 170 inbound trains per day (with up to 8,000 cars per day in total). The objective essentially minimizes a combination of the dwell time of the rail cars in the yard and delays to outbound trains. Running times of the algorithm are reported to be within 10 minutes.

Finally, simulation models for hump yard planning are described in $[15,16]$. The focus in these papers is not on optimizing the hump yard schedules, but rather in identifying bottlenecks in the existing infrastructure (i.e., the number of cars that can be handled) with existing scheduling strategies. 


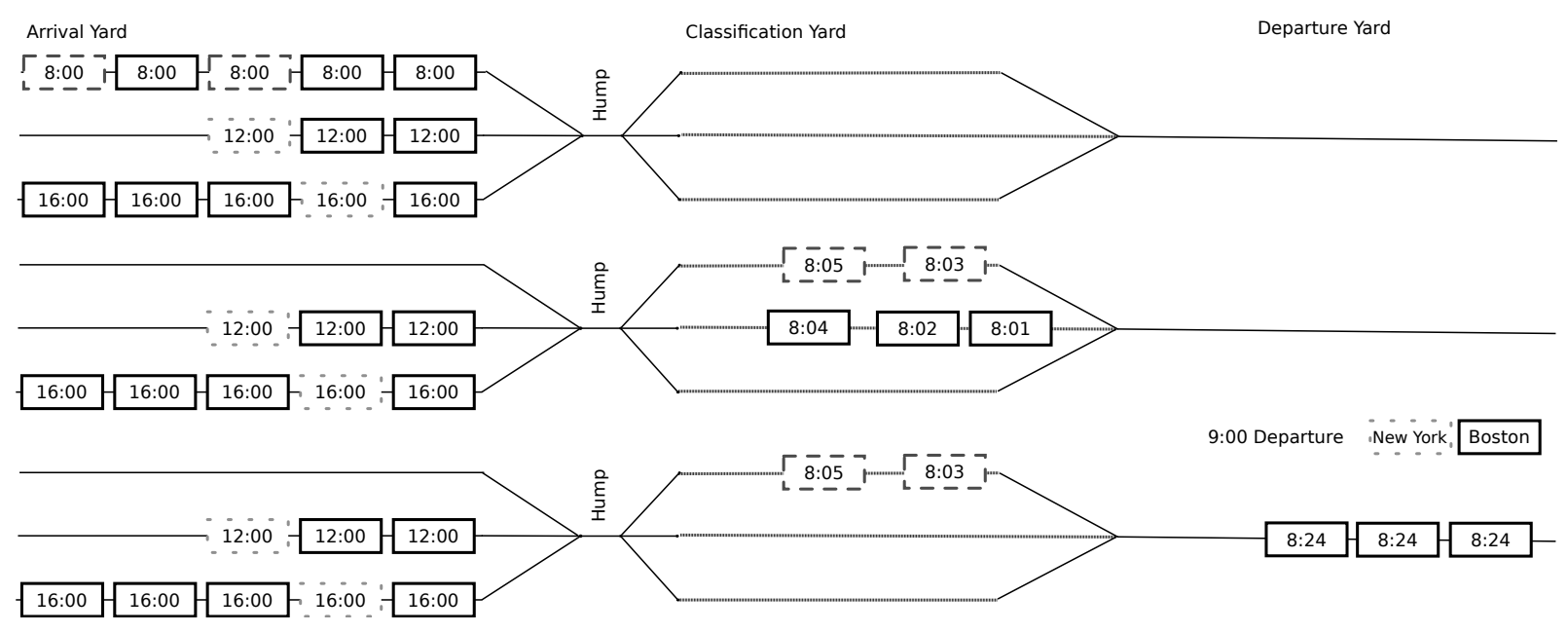

Figure 1: An example of a problem instance illustrating the railcars, the three different yards in three timesteps on the vertical axis. Railcars are represented as dashed, dotted and solid boxes corresponding to their assigned destinations (or block IDs). Assume one car can be processed by the hump every minute, and that the pullout time is 20 minutes. Three different inbound trains with distinct arrival times (8:00, 12:00 and 16:00) and one outbound train departing at 9:00 are shown. The top portion shows the initial state, where nothing is assigned. The middle portion shows an inbound train has been processed and bowl tracks have been assigned to the railcars. The bottom portion shows a cut has been pulled out and assigned to meet the departing train.

\section{Problem Description}

The considered problem has been introduced and defined by the Railway Application Section Competition 2014 [19]. In this section we present a standalone definition of the problem as well as introduce the notation we use throughout the paper. We reuse the notation and concepts of the original formulation to a great extent. An illustration of the problem mechanics is shown in Figure 1.

We assume that there is a set of inbound trains $\mathcal{I}$ arriving at the hump yard over a given time horizon. Each inbound train consists of a coupled, ordered sequence of railcars that will be separated at the hump in the yard. Each train $i \in \mathcal{I}$ arrives at the hump yard at a given time $a_{i}$, given in seconds from the start of the horizon. Note that this does not indicate the time at which it will be humped; a train can wait in the arrival yard as long as is necessary. The arrival yard is assumed to have infinite capacity. We denote the set of railcars by $\mathcal{C}_{i}$ for a given inbound train $i \in \mathcal{I}$. Naturally, the full set of cars to be processed is therefore $\mathcal{C}:=\bigcup_{i} \mathcal{C}_{i}$. Along with a length $l_{c}$, each car $c \in \mathcal{C}$ has a known block $I D$ (henceforth referred to as just block), denoted $b_{c}$. This indicates the car's next destination: for example BOS for Boston and $P H X$ for Phoenix. The set of all blocks is given by $\mathcal{B}$. As an example, Figure 2 indicates the composition of the first 10 inbound trains: in other words, the number of cars of different outbound blocks on each train. The bar chart provides a summary of the block counts only. On any given inbound train, the cars associated with a certain block are not usually in consecutive order, but distributed throughout the train. This random pattern is representative for the inbound trains in our dataset, i.e., we rarely see long sequences of cars with identical blocks. 
Inbound Train by Outbound Block

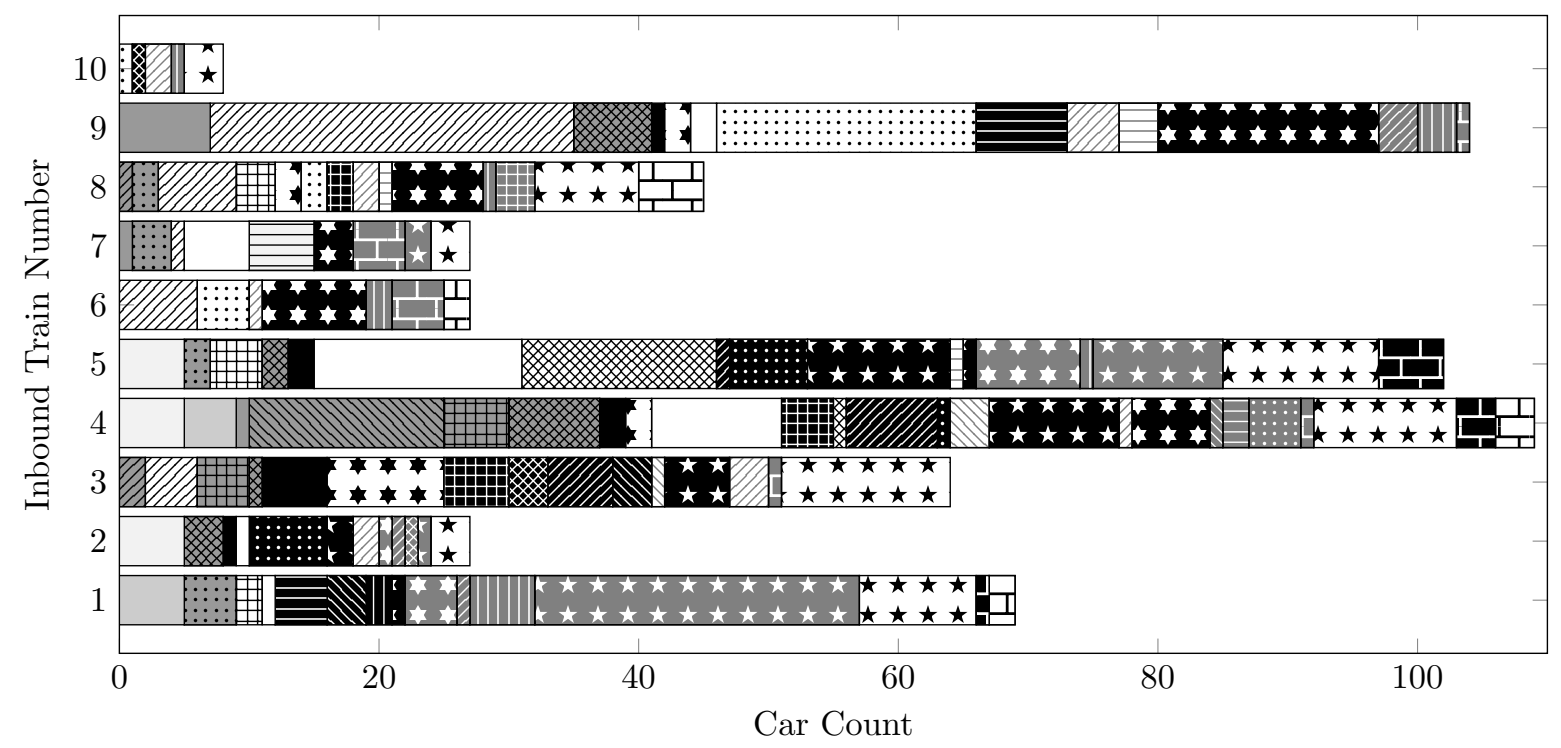

Figure 2: The different outbound blocks on each of the first 10 inbound trains. Different patterns correspond to different blocks.

Every inbound train $i \in \mathcal{I}$ must be processed, i.e., each of the cars $c \in \mathcal{C}_{i}$ must be decoupled, pushed over a hump, and moved to an available track in the classification bowl. Note that the process can be paused for a period, i.e., a partially decoupled inbound train can be stopped on the hump, e.g., if no track with sufficient space is available in the classification bowl.

It is assumed that the bowl consists of a set of parallel tracks $\mathcal{T}$, where each $t \in \mathcal{T}$ has a known length $\gamma_{t}$, and operates as a first-in-first-out queue. The tracks of the bowl are be used to sort the cars into lines of cars. A line is a sequence of cars of the same block and which are assigned to the same pullout (i.e., they appear on the same outbound train).

When humping a train several constraints must be respected. First, it is assumed two hump engines are available to perform the humping operations; one pulls cars from an inbound train to the hump, while the other is used to retrieve inbound trains from the arrival yard, i.e., bring them to the humping point. An inbound train's humping time refers to the time at which its first car is humped and two consecutive humpings must therefore be separated by some minimum duration. This duration is equal to either the minimum time required to hump all of the first train's cars (each car is assumed to take a constant time, $\lambda$ seconds, to hump), or the time required to retrieve the next inbound train from the arrival yard, again constant and equal to $\delta$ seconds, whichever is the longer. In addition, all of a train's cars must be humped before any other train can be humped, i.e, no train can be partially humped; it is considered to be an atomic operation.

Railcars that are coupled together are pulled from bowl tracks and appended to an awaiting outbound train. Each outbound train $o \in \mathcal{O}$ is scheduled to depart at the given time $d_{o}$ and has a maximum length $\eta_{o}$ of railcars that it can carry. An outbound train has a predetermined route through specific destinations (i.e., blocks) which is why the assigned railcars must conform to a standing order, that stipulates the order 
in which the railcar destinations (blocks) must appear on the outbound train.

Outbound trains are built in the departure yard using three available pullout engines. Pullout engines move so-called cuts of rail cars from the bowl to the departure yard. A cut simply refers to a sequence of lines that adhere to the standing order of the designated outbound train. Like hump engines, several restrictions exist for the pullout engines. First, pullouts from the same bowl track must be separated in time by a minimum duration to allow a smooth operation. Second, pullouts to the same outbound train must also be separated in time as multiple engines cannot build the same departure train simultaneously. Third, consecutive pullouts by the same engine must also be spaced by a minimum duration, corresponding to the time required to perform one job, which we denote $\rho$.

Finally, an outbound train can only be built within a certain time window of its scheduled departure time; e.g., for the considered problem, an outbound train can start to be assembled, at the earliest, 4 hours prior to its departure. However, we also consider an extension to the problem where departures can be delayed. Note that delaying a given outbound train will increase the dwell time for all cars assigned to that departure; however, it will also potentially reduce the dwell time of other rail cars that would otherwise miss the connection if it departed on time.

The objective of this problem is to determine the humping sequence of the inbound trains, which bowl track to use when humping each car, and the schedule for the pullout engines (i.e., how each outbound train should be built) such that all constraints are satisfied and the average dwell time of the cars in the yard is minimized. In other words, we need to determine an itinerary for each individual railcar (i.e., hump time, assigned bowl track, pullout time and outbound train), such that none of the constraints are violated.

\section{Modelling \& Methodology}

Given the problem's size and complexity, it is extremely difficult to construct a tractable mathematical model for the entire problem. Hence, we decompose the problem into three smaller, interdependent subproblems. which we term the Hump Sequencing Problem (HSP), the Block to Track Assignment Problem (BTAP), and The Pullout Allocation Problem (PAP), respectively. For the HSP and the PAP we describe MIP based optimization approaches, while we present a simple greedy heuristic for the BTAP. In this section we elaborate on each of these problems as well as the proposed methodology to solve them.

To set the context we provide a brief overview of the proposed methodology before going into specific details regarding each of the subproblems. Figure 3 illustrates the flow of the proposed approach. We begin by finding an arrival sequence for the inbound trains, e.g., using the HSP, and this remains fixed for the remainder of the algorithm. That is, after finding this processing sequence we never revise the humping order of the inbound trains. Cars are then iteratively humped into the bowl and assigned classification tracks using the BTAP. As soon as a car cannot be humped into the classification bowl, possibly due to a lack of space or no free tracks, the humping process is halted and pullouts are scheduled to make space in the bowl. Which pullouts to perform are decided by the PAP. Note that during an iteration the PAP and BTAP are not necessarily considering the bowl at the exact same time or time period. In general when the BTAP pauses at time $t$, e.g., due to lack of space or compatible tracks, the PAP will consider pullouts that occur before $t$. 


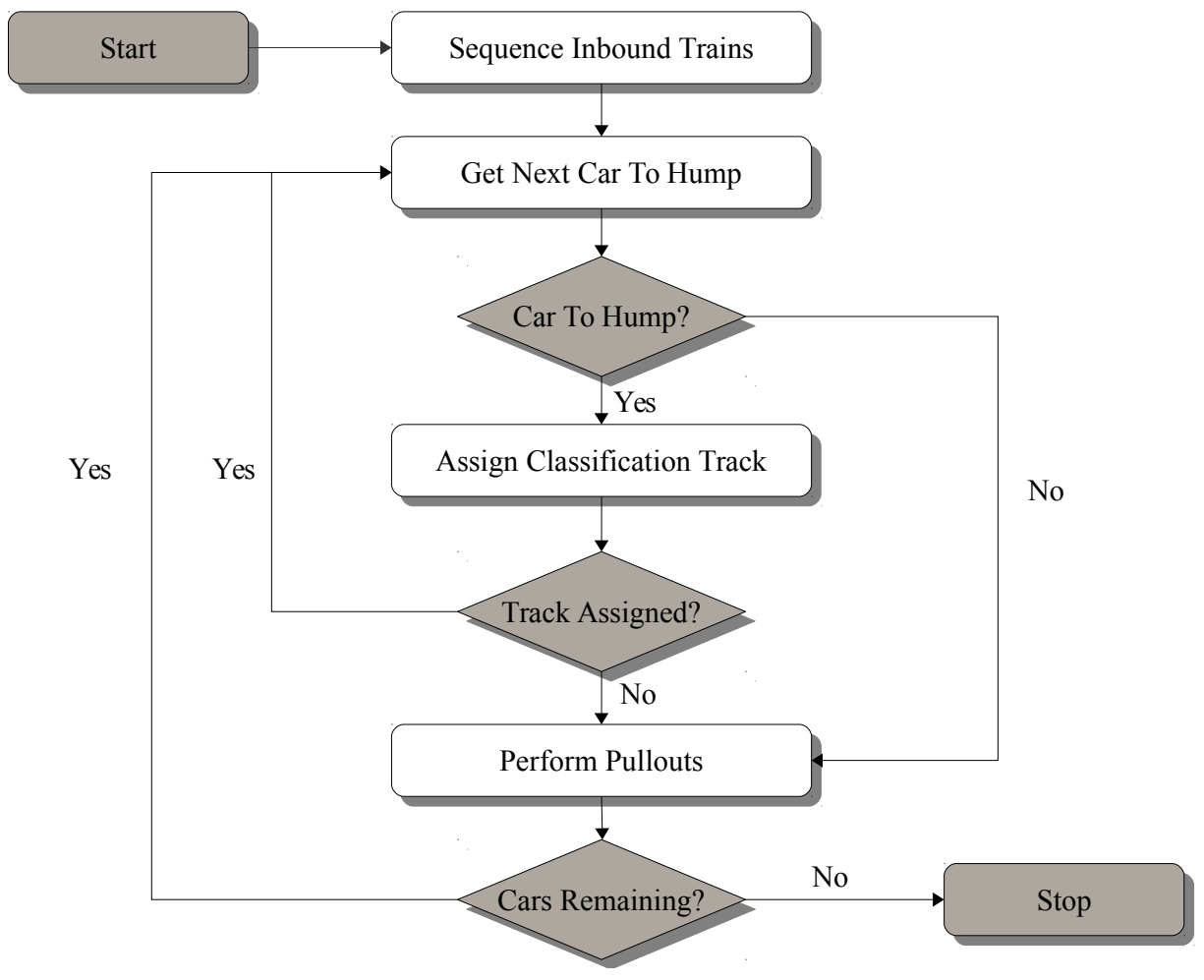

Figure 3: An overview of the proposed solution framework.

This process of humping cars and performing pullouts continues until there are no cars left to process. To provide a quality measure on the solutions found, in Section 4.1, we show how one can obtain lower bounds on the minimum average car dwell time. The lower bounds, albeit potentially weak, provide some sort of quality measure for the obtained solutions. Sections 4.2-4.4 are dedicated to the HSP, BTAP, and the PAP, respectively.

\subsection{Lower Bounds}

In order to verify the quality of the solutions produced by the matheuristic it is important to obtain a lower bound on the total dwell time. Here we describe two rather simple approaches for generating such bounds. Both bounds assume that outbound train departure times are fixed.

The first lower bound assumes that all outbound trains have infinite capacity, that all trains can be humped immediately, and that there are neither capacity nor ordering restrictions in the classification bowl. It does, however, respect the humping rate of the cars; the time at which any car is assumed to be available for a departure is a certain duration after the arrival time of the train it is on. This duration is the time needed to hump the cars ahead of it on the train.

The lower bound on average dwell time (in hours) is calculated as follows:

$$
L B=\frac{\left(\sum_{i \in \mathcal{I}} \sum_{c \in \mathcal{C}_{i}} d_{c}-a_{i}\right)}{3600 \cdot|C|},
$$


where the $d_{c}$ refers to the departure time of $c \in \mathcal{C}_{i}$. Here $d_{c}$ is simply the departure time of the earliest outbound train car $c$ can be assigned to such that $d_{c} \geq a_{i}+\lambda \cdot(n-1)$, where $n$ states the position of car $c$ in the sequence of cars on train $i$ and ranges from one to $\left|\mathcal{C}_{i}\right|$.

The second lower bound is calculated similarly, with the exception that outbound train capacity is taken into consideration. That is, $d_{c}$ denotes the earliest outbound train with available capacity car $c$ can be assigned to. The method processes cars ordered by their lengths and (for the ease of computation) allows fractional cars to be assigned to outbound trains. Naturally, the second bound is likely to be tighter than the first; however, the magnitude of this increase can provide insight into how restrictive the outbound train capacity is.

The bounds are calculated independent of the details of the classification bowl, and it will therefore not vary across the data sets we consider. Intuitively one would expect a more accurate bound to be higher in cases with fewer classification tracks since processing the cars in the bowl would likely require more time. Nevertheless, calculating the two bounds using Equation (1) yields values of 12.046 and 12.575 dwell hours in average, respectively.

Formulating and solving the second bound approach as a MIP, thereby removing fractionality, improves the bound to 12.591 hours at the cost of a $10^{4}$ factor increase in runtime.

\subsection{The Hump Sequencing Problem}

The hump sequencing problem entails identifying the best order in which to process the inbound trains. Depending on its block composition, it may or may not be critical to hump the cars on an inbound train into the bowl immediately. If, for example, the train is carrying cars for which the earliest outbound train is some time away, it may be preferable to hump another inbound train which arrives later but which carries cars for an earlier outbound train. Hence, simply processing inbound trains in their arrival order may result in some cars missing their earliest departure, and thus incurring unnecessary dwell time. We formulate this problem as a MIP and attempt to minimize the departure day of the final car to be processed. First, binary variables $x_{c o}$ are introduced and indicate whether or not car $c \in \mathcal{C}$ is assigned to outbound train $o \in \mathcal{O}_{c}$. $\mathcal{O}_{c}$ indicates the set of outbound trains to which car $c$ can be assigned, i.e., $\mathcal{O}_{c}=\left\{o: o \in \mathcal{O}, b_{c} \in \mathcal{B}_{o}\right\}$. A second set of binary variables $y_{i j}$ governs the sequencing order of inbound trains $i$ and $j \in \mathcal{I}$. In other words, the variable $y_{i j}$ takes the value one if train $i \in \mathcal{I}$ is humped before train $j \in \mathcal{I}$, and is zero otherwise. Note that for any two trains only one binary sequencing variable is required, and we use the arrival time of the trains to define a partial order. More specifically, we say $i \prec j$ if train $i \in \mathcal{I}$ arrives before train $j \in \mathcal{I}$. Finally, continuous variables $h_{i}$ are defined and represent the humping time of train $i$. A solution to this problem must respect several constraints. In particular, a minimum separation time must elapse between consecutive humpings, no car can depart before it has been humped, and the length of departing outbound trains must be respected. This problem does not consider the classification bowl nor the pullout engines explicitly and thus is expected to provide an optimistic solution; however, for problems with many classification tracks (i.e., more than $|\mathcal{B}|$ ) it should provide a good indication of the processing order. The full mathematical model is given below. 


$$
\begin{array}{cr}
\operatorname{minimize}: \sum_{c \in \mathcal{C}} \sum_{o \in \mathcal{O}_{c}} d a y(o) \cdot x_{c o}, & \\
h_{j} \geq h_{i}+\Delta_{i j}-M\left(1-y_{i j}\right) & \forall i, j \in \mathcal{I}, i \prec j, \\
h_{i} \geq h_{j}+\Delta_{j i}-M y_{i j} & \forall i, j \in \mathcal{I}, i \prec j, \\
\sum_{o \in \mathcal{O}_{c}} d_{o} x_{c o} \geq h_{i(c)}+i n d(c) \cdot \lambda & \forall c \in \mathcal{C}, \\
\sum_{c \in \mathcal{C}_{o}} l_{c} x_{c o} \leq \eta_{o} & \forall o \in \mathcal{O}, \\
\sum_{o \in \mathcal{O}_{c}} x_{c o}=1 & \forall c \in \mathcal{C}, \\
x_{c o} \in\{0,1\} & \forall c \in \mathcal{C}, o \in \mathcal{O}_{c}, \\
y_{i j} \in\{0,1\} & \forall i, j \in \mathcal{I}, i \prec j, \\
h_{i}, \geq 0 & \forall i \in \mathcal{I} .
\end{array}
$$

where $\operatorname{day}(o)$ gives the departure day of outbound train $o, i(c)$ gives the inbound train carrying car $c$, $\operatorname{ind}(c)$ gives the index of $\operatorname{car} c$ in $\mathcal{C}_{i}$, and $\mathcal{C}_{o}$ denotes the set of cars that can be assigned to outbound train $o \in \mathcal{O}$. The objective (2) minimizes the total number of days require to process all cars. Constraints (3) and (4) collectively ensure a minimum time separation between two consecutive humpings. Here $\Delta_{i j}=$ $\max \left(\lambda \cdot\left|\mathcal{C}_{i}\right|, \delta\right)$ which ensures a minimum separation time and enough time to hump cars of inbound train $i$, where $M$ is a sufficiently large number. In this case $M$ is equal to the latest arrival time of any train $i \in \mathcal{I}$. Constraints (5) stipulate that a certain time must elapse upon arrival before the car can depart on an outbound train. The maximum length of all outbound trains is enforced by constraints (6). This ensures that we cannot assign more rail cars to an outbound train than the outbound train can accommodate. Constraints (7) ensure that each car is assigned to exactly one departure. Finally, variable domains are given by constraints (8)-(10).

Due to the size of this model, it is solved using a kind of rolling time horizon based approach where each horizon has its own HSP and only considers a subset of arrivals. A rolling time horizon is justified in this context as any deviations from the arrival order when humping the inbound trains are likely to be local. For instance, it is quite unlikely trains will wait many days to be processed. By considering only a subset of arrivals at a time, we essentially limit the number of train ordering decisions that must be considered (thus reducing the number of $y_{i j}$ variables). This is because only the order of the trains in the subset under consideration can be modified. We therefore solve Model (2)-(10) as a sequence of smaller HSPs of the same form, where $\mathcal{I}$ is limited to $m$ trains. The subsets are generated based on the arrival order of the inbound trains. For example, the first HSP is solved using the first $m$ trains to arrive. To ensure a cohesive transition between the sequence of smaller HSPs, the sets of inbound trains used to define two successive HSPs intersect slightly. For example, if $m$ is 100, then after solving the first HSP, the solution found for the first 80 trains might be fixed. The second HSP then considers sequencing train 81 to train 180, and so on. Naturally, the capacity of the outbound trains is revised as we successively solve the HSPs due to any previous rail car assignments that have been fixed. In Section 5 this strategy 
is compared against the greedy approach of simply processing trains in their arrival order.

Finally, it is important to note that the solution to this problem might not be implementable in practice. Since the model does not consider classification tracks nor the pullout operations, there is no guarantee rail cars can actually leave on the departure to which they have been assigned in the HSP. The model is only used to provide an indication of hump sequencing order and is potentially quite optimistic.

\subsection{The Block-To-Track-Assignment Problem}

Whenever a rail car is humped, it must be assigned one of the classification tracks in the bowl. To assign a classification track we use a simple greedy heuristic. This procedure is as follows. If a line of the same block as that of the car being humped already exists in the bowl, and there is enough remaining capacity on the track, the car is humped to that track. Otherwise, a randomly selected "open track", i.e., an empty bowl track, is assigned. If neither option is possible, humping is momentarily paused, and pullouts are performed to create more space in the bowl.

Given the highly fragmented nature of the outbound blocks arriving on inbound trains, creating mixed tracks (i.e., classification tracks with possibly several different outbound blocks) would result in many short lines on the classification tracks. This in turn would lead to a greater number of pullouts being required to assemble the outbound trains. Thus, the greedy track allocation strategy ensures classification tracks are dedicated to specific blocks, possibly at the expense of more pauses in the humping of the rail cars. The performance of this simple heuristic strategy was compared to a more intelligent approach of assigning tracks based on outbound block volumes (classified as low, medium, or high based on number of cars). However, the latter, surprisingly, did not perform better.

\subsection{The Pullout Allocation Problem}

After humping has been performed in the framework, pullouts must be performed. Two pullout methods are developed and discussed. The first is a greedy approach (Greedy), while the second uses more sophisticated modelling techniques to better exploit the available pullout engine resources.

\section{Greedy Pullout}

The input for Greedy is a point in time, the bowl state at this time, and the next outbound train to process. Greedy sequentially processes outbound trains by departure time. For a given train $o \in \mathcal{O}$, it analyses all bowl tracks and tries to pull lines from tracks in the order specified by the departure's standing order. An overview of the method is shown in Algorithm 1.

The track with the longest available length is identified and pulled out (LongestTrack). The exact pullout time is determined by inspecting existing engine usage and pullouts. The LongestTrack subprocedure ensures that Greedy finds the earliest, feasible time for a pullout. After each pullout the time is advanced by the pullout time, since this is the minimum time required to do the next pullout. Although it is possible, the algorithm will never pull the same track more than once for the same departure. Note that we use an inner while loop in order to be able to pull out several tracks with the same block. 


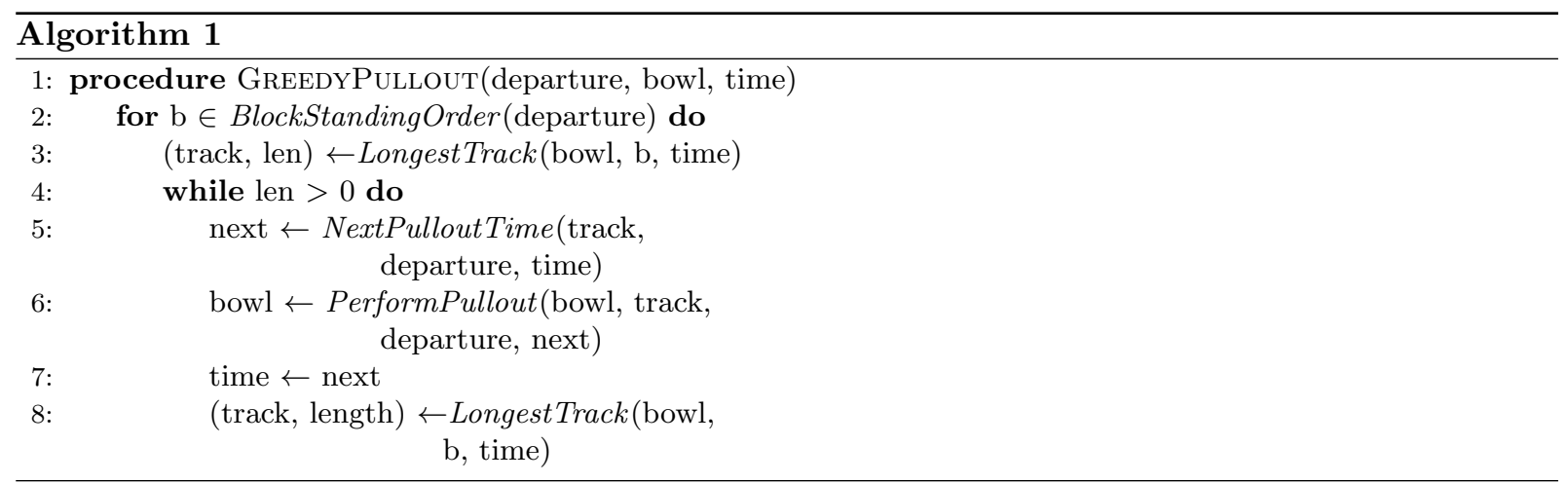

Railcars on bowl tracks

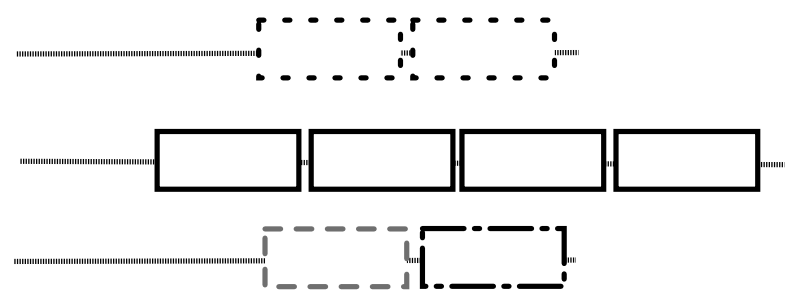

Naive Approach

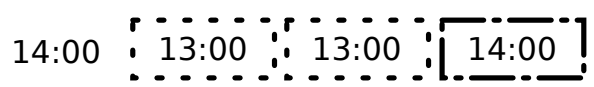

$14: 30$
Departures

14:00

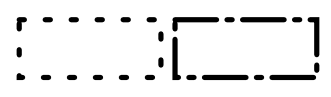

14:30

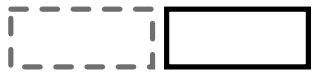

MIP Approach

14:00 $: 13: 00: 13: 00$

14:30

Figure 4: A comparison of the Greedy and MIP pullout approaches. In the top left portion the current bowl tracks are illustrated, and in the top right portion the scheduled departures are depicted. In this example one pullout engine is available and requires one hour per pullout job, and each departure can be built two hours in advance. The Greedy method processes the departures iteratively by departure time, thus in this example nothing is assigned to the second departures as the engine has been fully assigned up until 14:00. The MIP method, however, identifies a solution that shares the engines such that the total number of pulled railcars is maximized. 


\section{Pullout MIP}

One shortcoming of the Greedy method is its inability to share pullout engine resources and to consider what is beneficial or harmful for other outbound trains since it does not consider future consequences of local decisions. Here we propose a MIP-based pullout scheme that considers multiple outbound trains simultaneously. Figure 4 compares the methods using a simple example.

At any point in time each bowl track contains a set of lines. We will consider the line closest to the pullout end of any track; any line behind this will be left untouched. Therefore, at any time, each track $t \in \mathcal{T}$ can be mapped to a unique block and, consequently, a unique departure (and unique standing order therein). The mapping is not bijective since a standing order can be mapped to multiple compatible tracks. The input for this method is hence a point in time, time, and the corresponding bowl state. Based on this input and the instance data, we formulate a MIP model to identify which pullouts to perform. This MIP forms the basis of Algorithm 2.

For each track $t \in \mathcal{T}$ a list of possible pullout candidates $C_{t}$ is generated. All candidates for the same track differ in pullout time, effectively limiting how many cars can be pulled. The set of all candidates is denoted $C:=\bigcup_{t \in \mathcal{T}} C_{t}$.

A binary decision variable $x_{c}$ is introduced and indicates whether or not candidate $c \in C$ is selected. Some candidates cannot be selected simultaneously as this would create a standing order violation on the designated outbound train. We define set $C^{-} \subseteq C \times C$ to contain exactly these pairwise conflicts. $C^{-}$is determined in a preprocessing step.

If a candidate is selected we do not enforce all cars in the candidate to be pulled, only a subset. Therefore, for each track $t \in \mathcal{T}$ we monitor the total length of the cars pulled. To do this we introduce one continuous variable $y_{t}$ for each track $t \in \mathcal{T}$. Note that the decision variables $x_{c}$ and $y_{t}$ can lead to an infeasible decision as it may imply taking a percentage of some car. In practice we therefore leave the one fractional car behind. In addition, since all cars have individual lengths, these decision variables do not factor in the railcar by length ration. Optimality is thus no longer guaranteed, but we argue that a significant speed-up is achieved, through a simplified and smaller model, at a low cost. A fractional solution is only achieved when the outbound train has reached its capacity; this is rarely the case. Also note that obtaining an optimal solution here using a more precise model need not make the overall framework optimal.

We also define the set $\mathcal{T}_{o} \subseteq \mathcal{T}$, which gives the set of tracks containing lines for outbound train $o \in \mathcal{O}$. To accurately model the pullout movements two types of (time) interval sets are introduced. The first, $\Pi$, is the set of non-overlapping intervals generated by including all candidate pullout start and end times. An element, $\pi$, of this set is a subset of $C$ that overlaps with the interval. A similar second set, $\Omega_{o}$, contains all non-overlapping intervals generated by including all candidate pullout start and end times corresponding to a outbound train $o \in \mathcal{O}$. An element, $\omega$, of this set is a subset of $C$ that overlaps with the interval.

As mentioned in Section 3, outbound trains have a given capacity. Given the current bowl state and previous pullouts the remaining available length remCapo can be computed for all outbound trains. Finally, it is also given that we have a number, numEngines, of pullout engines available. The number of pullout engines being used, as a result of earlier choices, during interval $\pi \in \Pi$ is assumed to be usedEngines . . 
The pullout allocation MIP is as follows:

$$
\text { maximize: } \begin{aligned}
\sum_{t \in T} y_{t} \cdot o b j_{y} & \\
\sum_{c \in C_{t}} x_{c} & \leq 1 \\
x_{c}+x_{c^{\prime}} & \leq 1 \\
\sum_{t \in \mathcal{T}_{o}} y_{t} & \leq \text { remCap }_{o} \\
y_{\text {track }(c)} & \leq x_{c} \cdot \text { length }_{c}+M\left(1-x_{c}\right) \\
y_{t} & \leq \sum_{c \in C_{t}} x_{c} \cdot \text { lengt }_{c} \\
\sum_{c \in \omega} x_{c} & \leq 1 \\
\sum_{c \in \pi} x_{c} & \leq \text { numEngines }- \text { usedEngines }_{\pi} \\
x_{c} & \in\{0,1\} \\
y_{t} & \geq 0
\end{aligned}
$$

The objective is ideally to maximize the number of cars but as discussed we only model the number of feet pulled out. In this model we approximate the number of cars by setting $o b j_{y}$ to the average number of cars per feet. This approximation works well due to the fact that the capacity of most outbound trains is not binding. And, in any case, all cars must be pulled out sooner or later.

The first set of constraints (12) ensure at most one candidate is selected per track. Constraints (13) guarantee that pullouts respect the outbound train's standing order by prohibiting conflict pairs. The remaining capacity of an outbound train must be respected; this is the purpose of Constraints (14). Constraints (15) link the pullable length of the track to the selected candidate decision, while (16) makes sure that zero length is pulled from a track if no candidates from the track are selected. Constraints (17) ensure candidates selected for the same departure, $o$, respect a minimum distance. This is modeled by making sure that one candidate can remain active (in a pullback process) in the same interval for the same departure. Similarly constraints (18) ensure that at most numEngines engines are used concurrently in every period. Finally, variable domains are given by (19) and (20).

\section{Framework Integration}

This MIP-based procedure is called every $x$ minutes at time $t=t_{\text {last }}+x$, where $x$ is set to an appropriate value set in the tuning phase. The pullout MIP identifies the best set of pullouts to be performed; however, not all are actually performed by the overall framework since the model only considers partial information based on the current state of the bowl. Ideally, one only wants to perform pullout decisions that will definitely not change in later iterations,. In our case, this corresponds to every decision made before the last hump time or before time $t$. An overview of the algorithm is given in Algorithm 2. Using a discretization step all candidate pullouts after time within the build window of the departures are generated. Next the list of candidates is filtered. All candidates that violate standing orders due to existing pullouts in 
the bowl are removed. Candidates that violate the separation constraint (of past assignments) on the corresponding track are removed. Candidates that violate the separation constraint (of past assignments) on the departure are also removed. The generated candidate pullout events and existing engine usage are analyzed and the available engine capacity is stored for all relevant time periods.

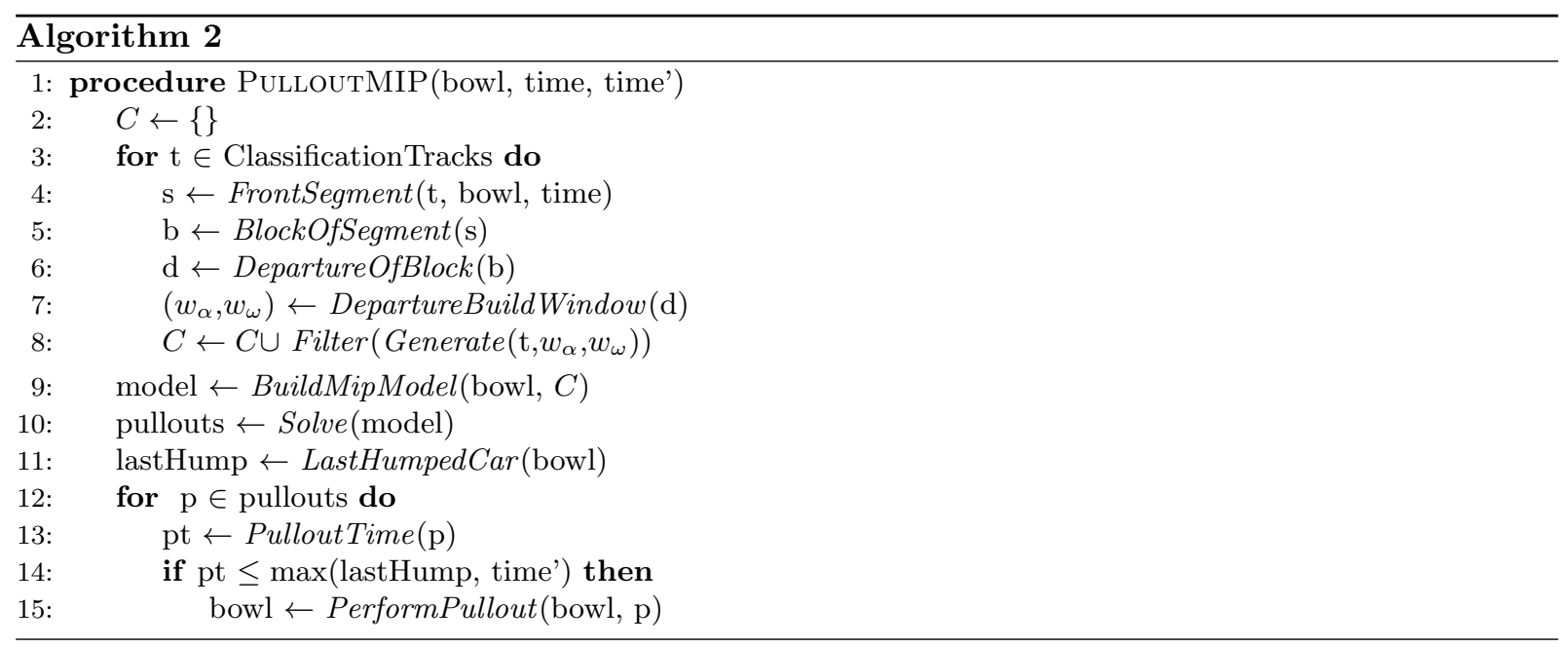

An example illustrating the pullout process in the solution framework is shown in Figure 5. For the sake of argument we make a few simplifications. First, assume that every outbound train only has a standing order of one block type. Second, assume that only one pullout engine exists and requires one hour to perform one pullout. Third, assume that a outbound train can be built two hours in advance. Finally, assume that all rail cars are of equal length. The example shows the relation between the decisions made by the MIP (Algorithm 2) and the decisions actually implemented by the solution framework. Only a subset of the the MIP decision are adopted by the framework as it only considers partial information. Note, in Figure 5 at 12:00 the MIP recommends pulling two blue rail cars at 12:00-13:00 but in the next iteration this decision has been changed (to pull three red rail cars instead) as more rail cars have arrived in the bowl. Also note, in the second iteration, that the decision to pull three red rail cars at 13:00-14:00 is changed to pull four rail cars from the same track in the third iteration.

This approach is superior to the Greedy approach as it considers interdependencies between multiple departures. Although the method operates on partial future information, depending on how much is in the bowl, it can still consider future consequences of pullouts to some extent. Solving the MIP model using a commercial solver allows us to find near optimal solutions very quickly in practice. This benefit is, however, also a liability since the runtime overhead of using a general purpose solver must be paid. The model has to be built and solved many times - in some cases building the model is more expensive than solving it. For practical reasons, the number of generated candidates is limited or discretized, and therefore the model is unable to gain a fine-grained control of the pullouts.

Finally, we mention that this approach assumes that only one line can be pulled out simultaneously. The model should ideally consider the possibility of pulling multiple lines on a particular track; however, given the limited number of lines allowed simultaneously in the bowl, this did not seem to be a critical concern. The proposed model can be extended without much difficulty to handle multiple lines. 
Implemented

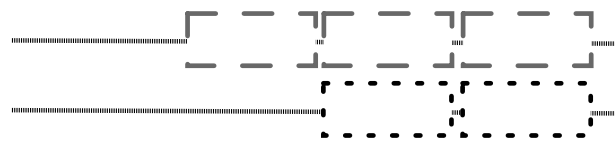

11:00-12:00

11:00-12:00

Departure schedule

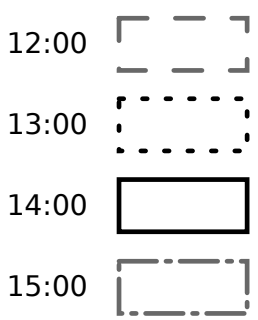

13:00-14:00

12:00-13:00

12:00-13:00

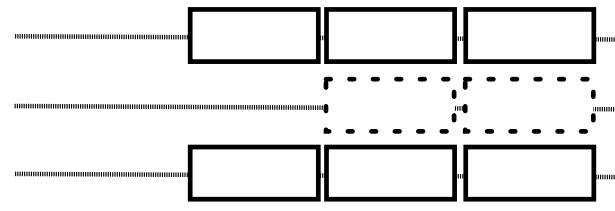

14:00-15:00

$14: 00$

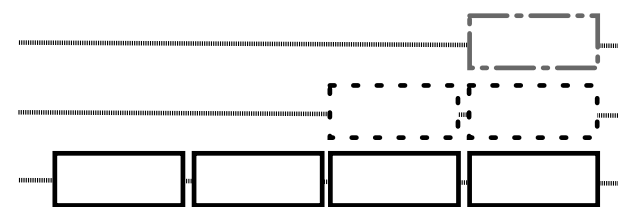

13:00-14:00
13:00-14:00

Figure 5: An illustration of three pullout iterations in the solution framework. The bowl contains three classification tracks. The boxes represent rail cars where the target departures (blocks) are represented using different types of strokes. Here, the iterations occur every hour which means that more units have been processed over the hump in subsequent iterations. The MIP column shows the pullout decisions made in each iteration, and the Implemented column shows which pullouts were implemented. Finally to the right, the departure schedule is listed. In this example, three pullouts are performed. At 11:00-12:00 and 12:00-13:00 the top track is pulled out with respectively three green and red rail cars. At 13:00-14:00 the bottom track is pulled out with four red rail cars. 


\begin{tabular}{lrr}
\hline Inbound trains & 702 & \\
Rail cars & 52,247 & \\
Rail cars length & $3,049,094$ & feet \\
Horizon & 42 & days \\
\hline Min train length & 55.0 & feet \\
Max train length & $9,200.0$ & feet \\
Average train length & $4,343.4$ & feet \\
\hline Min train cars & 1 & \\
Max train cars & 161 & \\
Average train cars & 74.4 & \\
\hline Min distinct blocks & 1 & \\
Max distinct blocks & 32 & \\
Average distinct blocks & 15.3 & \\
\hline
\end{tabular}

Table 1: Statistics on the instance inbound trains.

\begin{tabular}{rrr}
\hline Instance No & Tracks & Total Length \\
\hline 1 & 58 & 122,489 \\
2 & 50 & 109,853 \\
3 & 42 & 96,354 \\
\hline
\end{tabular}

Table 2: List of data instances. The columns show the instance number, number of classification tracks in the bowl, and the total length (in feet) of tracks.

\section{Computational Results}

In this section we discuss and benchmark our algorithm by incrementally enabling the more advanced features. For each experiment we report a few key performance indicators. All runs are performed multiple times in order to examine the expected average performance and expected deviation; the variation is due to the heuristic used for the BTAP. We consider the data instances for the HYBA problem introduced by in the RAS Problem Solving Competition in 2014 [19]. Tables 1-3 respectively summarize the instance characteristics, differences and parameters.

First, we evaluate the framework performance using the Greedy pullout algorithm processing inbound trains on the hump by arrival order. The results are shown in Table 4 . The results show that we can solve the instances within 2 seconds achieving a optimality bound of $30-35 \%$ for the first two instances, but around a staggering $185 \%$ for the third instance. The deviation between runs is very low for the first two (less than 0.1 standard deviation in hours); however, it spans $140.75 \%-287.68 \%$ for the last instance.

\begin{tabular}{lrl}
\hline Hump engines & 2 & \\
Pullout engines & 3 & \\
Time between train humping & 20 & Minutes \\
Humping rate & 30 & Seconds \\
Pullout time per job & 20 & Minutes \\
Start build time offset & 4 & Hours \\
\hline
\end{tabular}

Table 3: List of instance parameters. 


\begin{tabular}{rrrrrrrrr}
\hline & & \multicolumn{7}{c}{ Dwell (hours) } \\
\cline { 2 - 7 } No & Time & Arrival & Bowl & Departure & Total & Max & Lines & Gap \\
\hline 1.0 & 1.7 & 0.59 & 12.35 & 3.43 & 16.38 & 94.7 & 61.0 & $30.2 \%$ \\
2.0 & 1.4 & 0.92 & 12.52 & 3.47 & 16.90 & 87.8 & 53.5 & $34.4 \%$ \\
3.0 & 1.2 & 19.65 & 12.69 & 3.51 & 35.85 & 91.4 & 44.8 & $185.1 \%$ \\
\hline
\end{tabular}

Table 4: Results using a greedy humping and pullout strategy. The columns respectively show the instance number, average runtime in seconds, different average dwell time averages in hours, the average maximum no of concurrent lines in the bowl, and finally the relative optimality gap.

\begin{tabular}{|c|c|c|c|c|c|c|c|c|}
\hline \multirow[b]{2}{*}{ No } & \multirow[b]{2}{*}{ Time } & \multicolumn{5}{|c|}{ Dwell (hours) } & \multirow[b]{2}{*}{ Lines } & \multirow[b]{2}{*}{ Gap } \\
\hline & & Arrival & Bowl & Departure & Total & Max & & \\
\hline 1.0 & 28 & 0.56 & 11.39 & 1.22 & 13.17 & 129.9 & 61.0 & $4.74 \%$ \\
\hline 2.0 & 273 & 0.57 & 11.29 & 1.35 & 13.21 & 117.6 & 54.2 & $5.07 \%$ \\
\hline 3.0 & 338 & 1.42 & 10.92 & 1.99 & 14.33 & 76.6 & 45.5 & $13.92 \%$ \\
\hline
\end{tabular}

Table 5: Results using a greedy humping method and the MIP pullout method. The columns respectively show the instance number, average runtime in seconds, different average dwell time in hours, the average maximum no of concurrent lines in the bowl, and finally the relative optimality gap.

We do not see much improvement nor loss when activating low or high volume track selection for the BTAP, mentioned in Section 4.3. Runtime remains unchanged, but a benchmark showed that only the last data set is improved up to 2.4 average dwell hours.

The biggest improvement was observed when using the MIP method for the PAP instead of Greedy. The results are summarized in Table 5. The benchmark reports a significant improvement in average/bound for all instances. The last instance is, however, still around ten percentage points above the other two. As expected, the runtime is increased, from a few seconds to a few minutes. A noticeable increase in maximum dwell time can be observed compared to the previous benchmark, but the average maximum number of lines is not changed significantly. This is to be expected since the MIP pullout approach does not process the bowl on a first-come first-served fashion.

Finally we benchmark the performance of using the PAP MIP together with the HSP MIP method. The results are shown in Table 6. A consistent improvement of 0.06 to 0.09 average hours was observed, i.e., roughly 5000 dwell hours. It is observed that this setting improves the average dwell time at the bowl and departure yards, while achieving a slightly increased dwell time at the arrival yard. The HSP MIP method finds a better processing order of the arriving trains, at the cost of processing them more quickly by arrival order. This improvement does, however, come at the cost of approximately twice the runtime. The maximum dwell time and average maximum line usage show no noticeable change. A histogram showing the rail car dwell hour distribution, of one solution, is plotted in Figure 6. The majority of the rail cars leave the yard within 25 hours of arrival. Very few rail cars stay in the yard more than 50 hours. The solutions of all instances show a similar shape to Figure 6. Compared to the first two instances, the last instance contains fewer rail cars with 0-5 hours dwell time and an increased number of rail cars with more than 25 hours dwell time. 


\begin{tabular}{|c|c|c|c|c|c|c|c|c|c|c|c|}
\hline \multirow[b]{2}{*}{ No } & \multicolumn{3}{|c|}{ Time (s) } & \multicolumn{5}{|c|}{ Dwell (hours) } & \multirow[b]{2}{*}{ Lines } & \multirow[b]{2}{*}{ Gap (\%) } & \multirow[b]{2}{*}{ Gap } \\
\hline & Total & Hump & Pullout & Arrival & Bowl & Departure & Total & $\operatorname{Max}$ & & & \\
\hline 1.0 & 522 & 238 & 280 & 0.66 & 11.25 & 1.19 & 13.09 & 137.53 & 61.1 & $4.09 \%$ & 0.51 \\
\hline 2.0 & 507 & 238 & 266 & 0.67 & 11.12 & 1.33 & 13.12 & 133.77 & 54.3 & $4.37 \%$ & 0.55 \\
\hline 3.0 & 570 & 237 & 330 & 1.50 & 10.82 & 1.95 & 14.27 & 73.32 & 45.3 & $13.47 \%$ & 1.69 \\
\hline
\end{tabular}

Table 6: Final results obtained using the MIP method for both hump sequencing and pullouts. The columns respectively show the instance number, average runtime in seconds, different average dwell time in hours, the average maximum number of concurrent lines in the bowl, and finally the relative and absolute gap to our lower bounds.

\begin{tabular}{|c|c|c|c|c|c|c|c|}
\hline \multirow[b]{2}{*}{ Scenario } & \multicolumn{3}{|c|}{ Runtime (s) } & \multirow[b]{2}{*}{ Dwell } & \multirow[b]{2}{*}{ Max } & \multirow[b]{2}{*}{ Lines } & \multirow[b]{2}{*}{ Change } \\
\hline & Total & Hump & Pullout & & & & \\
\hline Long tracks & 414.2 & 247.7 & 150 & 12.96 & 71.0 & 45.0 & -0.14 \\
\hline Many engines & 662.0 & 245.5 & 413.0 & 13.08 & 138.2 & 60.9 & -0.02 \\
\hline Many Lines & 608.7 & 255.0 & 349.6 & 13.10 & 138.6 & 61.1 & -0.00 \\
\hline Long trains & 429.4 & 150.2 & 275.9 & 12.47 & 47.6 & 57.8 & -0.63 \\
\hline
\end{tabular}

Table 7: Results for the what-if scenario benchmarks. The columns respectively show the scenario name, average runtime in seconds, average dwell time in hours, maximum dwell time of an individual car, the average maximum number of concurrent lines in the bowl, and finally the change in average dwell-time compared to the normal instance.

\subsection{What-If Scenarios}

We perform a second line of what-if scenarios in order to identify the current bottlenecks of the data sets. In isolation we change the parameters and data instances in order to achieve different results. All changes are based on the first original instance, i.e, the least restrictive instance with most classification tracks.

The complete set of scenarios and results are listed in Table 7. In the first scenario, we increase the size of all tracks in the bowl. In the second we add more pullout engines. In the third we allow a very high number of lines. In the fourth we allow longer outbound trains.

The experiments show that the average dwell is improved most by adding longer tracks or longer outbound trains. Adding more pullout engines or allowing more concurrent lines in the bowl does not have a significant impact.

\subsection{Delayed Departures}

The MIP-based lower bound method from Section 4.1 can without much difficulty be extended to allow departure delays. In order to make the altered model tractable, we make alterations that allow an outbound train to select between departure times in a discrete set. The optimal solution is therefore a guess (or an optimistic guess) of the average dwell time when allowing departures.

Due to the discretization, this is not a true lower bound when considering delays; however, it is a good indication that one can expect improvements by considering delays for outbound trains. In our test we allow delays of up to 380 minutes with 20 minutes discretization. This results in a lower bound of 10.10 
average dwell hours. This significant reduction suggests that large savings can be achieved by allowing departures to be delayed. We note that roughly half of all outbound trains were delayed in this lower bound solution.

Finally, we benchmarked the result of allowing up to 2 hours delay in our solution methods, thus getting a real solution instead of a bound. These settings generate average dwell hours of 12.31, 12.33, and 12.42 the three datasets. Again, a significant improvement, especially for the last instance.

\section{Conclusions}

In this paper we consider the HYBA problem. We propose a heuristic framework which decomposes the problem into three interdependent subproblems. A version of the algorithm in which we consider greedy strategies for the humping and pullout process obtains acceptable solutions within two seconds. A second version, in which the humping and pullout strategies are solved using MIP models, obtains solutions that are significantly better; however, it does take substantially more time. The runtime is, however, still very reasonable considering the length of the planning horizon. The solutions obtained have a proven optimality gap of a few percent. An additional experiment shows that significant improvements can be obtained by allowing outbound trains to be delayed.

In addition to solving the HYBA problem the proposed heuristic method can be used to estimate the effect of infrastructure or equipment investments. Several what-if scenarios are considered and the results show that the studied data instance can benefit from longer outbound trains and additional track-length in the classification yard. However, allowing more concurrent lines in the bowl or using additional pullout engines does not make a significant difference.

Simple methods have been proposed for finding lower bounds for the problem. The results show that the lower bounds give good estimates for the two first instances. The methods do not take the bowl tracks into account, which explains why a weaker bound is achieved for the last instance. Promising directions for future research include strengthening this lower bound calculation to reflect the limitations of fewer

bowl tracks as well as integrating certain components of the algorithm. In particular, one idea could be to allocate a set of rail cars to bowl tracks when humping a specific car, as opposed to the current approach of greedily allocating each individual rail car a track when it is being humped. Finally, having the ability to dynamically adjust the humping sequence may also yield further improvements.

\section{References}

[1] R.K. Ahuja, K.C. Jha, and J. Liu, Solving real-life railroad blocking problems, Interfaces 37 (2007), 404-419.

[2] L.D. Bodin, B.L. Golden, A.D. Schuster, and W. Romig, A model for the blocking of trains, Transportation Res Part B: Methodological 14 (1980), 115 - 120.

[3] M. Bohlin, F. Dahms, H. Flier, and S. Gestrelius, Optimal freight train classification using column generation, ATMOS, Vol. 25 of OASICS, Schloss Dagstuhl - Leibniz-Zentrum fuer Informatik, 2012, pp. $10-22$. 

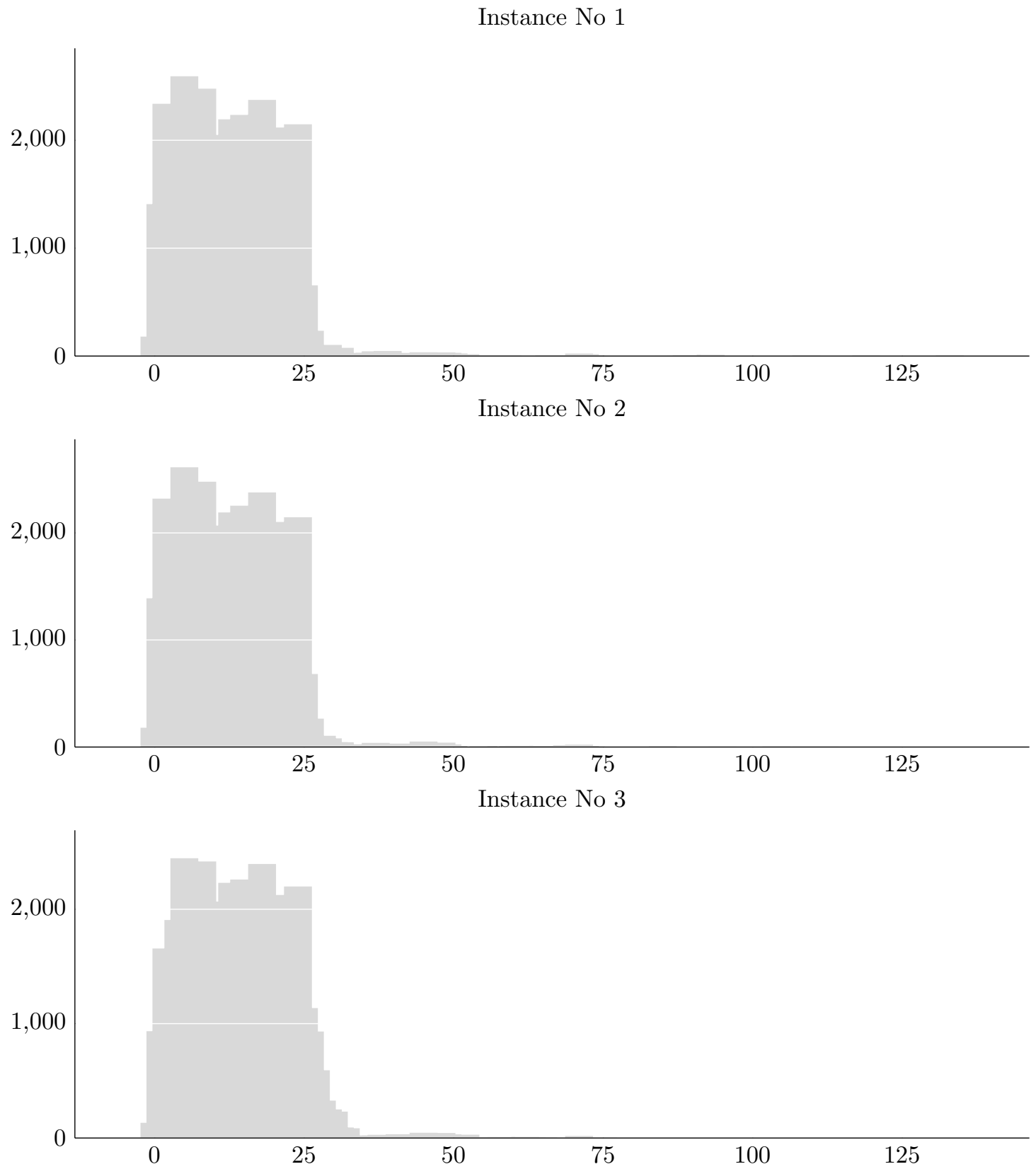

Figure 6: Histogram of rail car dwell times. Histogram shows the number of rail cars grouped by dwell hours. 
[4] M. Bohlin, F. Dahms, and S. Gestrelius, Optimisation of simultaneous train formation and car sorting at marshalling yards, Report 2013-013, Operations Research, RWTH Aachen University, Jul 2013.

[5] M. Bohlin, S. Gestrelius, F. Dahms, M. Mihalák, and H. Flier, Optimized shunting with mixed-usage tracks, Technical report 2013-12-19, Swedish Institute of Computer Science, 2013.

[6] N. Boysen, M. Fliedner, F. Jaehn, and E. Pesch, Shunting yard operations: Theoretical aspects and applications, Eur J Oper Res 220 (2012), 1 - 14.

[7] D. Briskorn and F. Jaehn, A note on "Multistage methods for freight train classification", Networks 62 (2013), 80-81.

[8] E. Dahlhaus, P. Horak, M. Miller, and J.F. Ryan, The train marshalling problem, Discr Appl Math 103 (2000), $41-54$.

[9] J.R. Dirnberger and C.P.L. Barkan, Lean railroading for improving railroad classification terminal performance: Bottleneck management methods, Transportation Res Record: J Transportation Res Board 1995 (2007), 52 - 61.

[10] M. Gatto, J. Maue, M. Mihalák, and P. Widmayer, "Shunting for dummies: An introductory algorithmic survey," Robust and online large-scale optimization, R. Ahuja, R. Möhring, and C. Zaroliagis (Editors), Springer, 2009, Vol. 5868 of Lecture Notes in Computer Science, pp. 310-337.

[11] S. He, R. Songa, and S.S. Chaudhry, An integrated dispatching model for rail yards operations, Comput \& Oper Res 30 (2003), 939 - 966.

[12] R. Jacob, P. Marton, J. Maue, and M. Nunkesser, Multistage methods for freight train classification, Proc 7th Workshop Algorithmic Approaches Transportation Modeling, Optim, Syst (ATMOS'07), Schloss Dagstuhl, Internationales Begegnungszentrum für Informatik, Nov 2007, pp. 158-174.

[13] E.R. Kraft, Priority-based classification for improving connection reliability in railroad yards. part I of II: Integration with car scheduling, Transportation Res Forum (2002), 93-105.

[14] E.R. Kraft, Priority-based classification for improving connection reliability in railroad yards. part II of II: Dynamic block to track assignment, Transportation Res Forum (2002), 107-119.

[15] E. Lin and C. Cheng, Yardsim: A rail yard simulation framework and its implementation in a major railroad in the U.S., Winter Simulation Conference, WSC, 2009, pp. 2532-2541.

[16] E. Lin and C. Cheng, Simulation and analysis of railroad hump yards in North America, Winter Simulation Conference, WSC, 2011, pp. 3715-3723.

[17] J. Maue, On the problem of sorting railway freight cars: An algorithmic perspective, Ph.D. Thesis, ETH Zurich, Göttingen, Germany, July 2011.

[18] H.N. Newton, C. Barnhart, and P.H. Vance, Constructing railroad blocking plans to minimize handling costs, Transportation Sci 32 (1998), 330-345. 
[19] Railway Applications Section, Institute for Operations Research and the Management Sciences (INFORMS), Railroad hump yard block-to-track assignment, webpage. www.informs.org/Community/ RAS. 